Reprod. Nutr. Dévelop., 1987, 27 (1 В), 235-236.

\title{
Evolution du transit digestif au cours de la période de sevrage chez le veau
}

\author{
J. P. LALLĖS, C. PONCET
}

Laboratoire de la Digestion des Ruminants, I.N.R.A. Theix 63122 Ceyrat, France.

Summary. The mean retention time (MRT) of feed was measured during weaning in calves. The MRT of hay (50 hours) in the whole digestive tract was not modified by weaning while the MRT of concentrate decreased from 50 to 40 hours between 9 and 20 weeks of age. The rumen MRT increased from 40 to $60-70 \%$ of the total MRT whereas the MRT in the small and large intestines decreased.

Le temps de rétention moyen (TRM) des résidus alimentaires indigestibles dans l'ensemble du tube digestif (TD) du jeune ruminant consommant du foin ne semble pas varier au cours du sevrage (Hodgson, 1971). L'évolution du TRM dans les différents compartiments digestifs n'ayant pas été précisée, nous l'avons étudiée au cours d'une expérience de mesure de flux digestifs pendant le sevrage. Ces résultats préliminaires (5 veaux sur 12) n'ont pas été traités statistiquement.

Matériel et méthodes. 5 veaux mâles frisons, fistulés à l'âge de 3 semaines, ont commencé à ingérer (ad libitum entre les périodes de mesure) un régime solide comprenant $20 \%$ de foin haché et $80 \%$ d'aliment concentré (amidon de maïs-tourteau de soja ou amidon de maïs-pois) distribués respectivement en 2 et 8 repas par jour, à l'âge de 7 semaines. lis ont été sevrés à 10 semaines. Les 3 périodes de mesure du TRM de chaque constituant de la ration ont eu lieu aux âges de 9-10, 15-16 et 19-20 semaines. Les aliments marqués (ytterbium-169 sur le foin, cérium-141 sur l'aliment concentré) (Ellis et Beever, 1984) ont été distribués simultanément (dose unique) au début d'un repas. Les courbes d'excrétion fécale des marqueurs ont été analysées selon le modèle de Grovum et Williams (1973).

Résultats et discussion. Le TRM du foin dans l'ensemble du TD n'évolue pas au cours du sevrage (tabl. 1), ce qui confirme les résultats de Hodgson (1971). Le TRM de I'aliment concentré, identique à celui du foin avant le sevrage ( $\simeq 50 \mathrm{~h}$ ), diminue de $20 \%$ entre 9 et 20 semaines d'âge. Le développement des

TABL. 1. - Evolution du temps de séjour des 2 constituants de la ration dans l'ensemble du tube digestif des veaux.

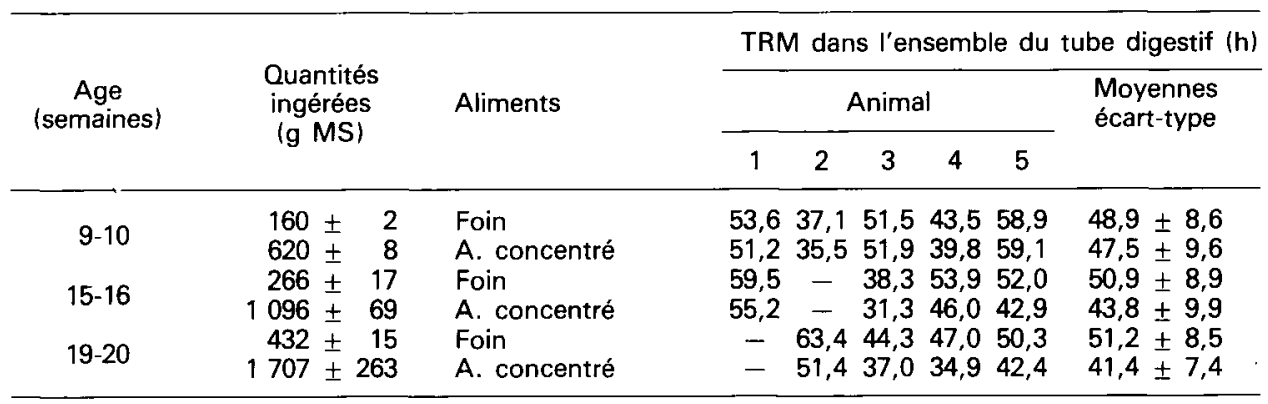


fonctions de rumination et de salivation ainsi que de la motricité du rumen a dû conduire à une ségrégation progressive des 2 constituants de la ration comparable à celle observée chez le ruminant adulte (Eng et al., 1964).

Les TRM du foin et de l'aliment concentré dans le rumen, exprimés en $\%$ des TRM respectifs, passent respectivement de 44 à 68 et de 45 à $61 \%$ entre 10 et 20 semaines d'âge (fig. 1). Ceci s'explique essentiellement par l'augmentation concomitante du volume du contenu de rumen. En contrepartie, les TRM du foin et de l'aliment concentré dans le caecum-colon passent de 22 à $10 \%$ des TRM totaux. La contribution de l'intestin grêle au TRM total passe de 33 à $20 \%$ pour le foin alors qu'elle varie peu pour l'aliment concentré $(30 \%$ ) (en valeur absolue, le temps de transit des 2 aliments est identique et varie de 15-16 h à 10-12 h durant cette période). Cette accélération du transit intestinal peut résulter de l'augmentation de l'activité motrice irrégulière observée par Ruckebusch et Bueno (1973).

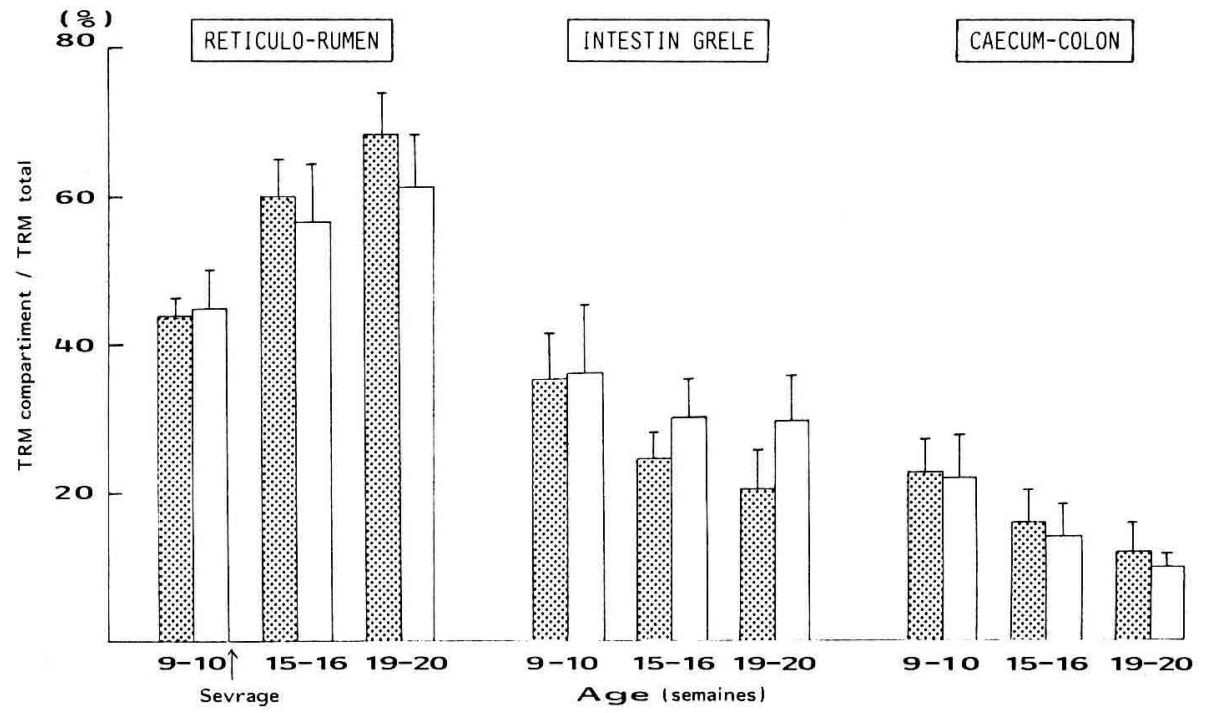

FIG. 1. - Evolution des TRM des 2 constituants de la ration dans chaque compartiment du tube digestif des veaux (Foin $\square$; Aliment concentré $\square$ ).

Le sevrage affecte peu le TRM total des résidus alimentaires dans le T.D. du veau, mais le temps de séjour dans le rumen semble s'accroître au détriment de ceux concernant l'intestin grêle et le caecum-colon.

Ellis W. C., Beever D. E., 1984. In P. M. Kennedy, Techniques in particle size analysis of feed and digesta in ruminants. Can. Soc. Anim. Sci., Edmonton, 167.

Eng K. S., Riewe M. E., Craig J. H., Smith J. C., 1964. J. anim. Sci., 23, 1129-1132.

Grovum W. L., Williams V. J., 1973. Br. J. Nutr., 30, 313-329.

Hodgson J., 1971. Anim. Prod., 13, 449-460.

Ruckebusch Y., Bueno L., 1973. J. Nutr., 30, 491-499. 